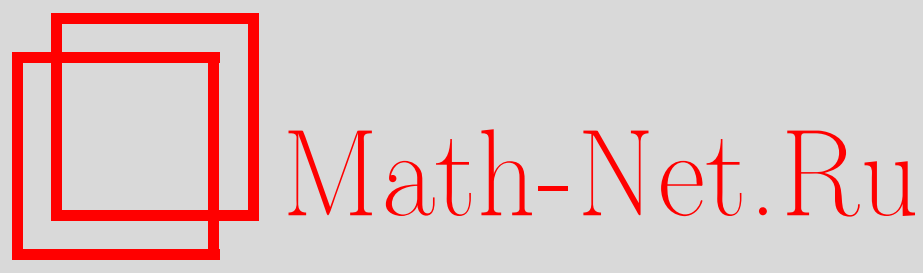

Ф. Бланк, Условная оптимальная оценка сверху аргумента дзета-функции Римана на критической прямой, Совр. пробл. матем., 2016, выпуск 23, 24-34

DOI: https://doi.org/10.4213/spm59

Использование Общероссийского математического портала Math-Net.Ru подразумевает, что вы прочитали и согласны с пользовательским соглашением http://www . mathnet.ru/rus/agreement

Параметры загрузки:

IP : 54.198 .55 .26

26 апреля 2023 г., 18:34:35

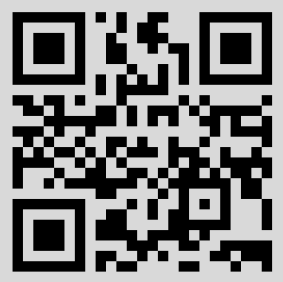




\title{
Условная оптимальная оценка сверху аргумента дзета-функции Римана на критической прямой
}

\author{
Ф. Бланк \\ Haute École d'Ingénierie et de Gestion du Canton de Vaud, Switzerland \\ philippe.blanc@heig-vd.ch
}

\section{1. Введение}

Пусть $\zeta$ есть дзета-функция Римана, определяемая при $\operatorname{Re}(s)>1$ как

$$
\zeta(s)=\sum_{n=1}^{\infty} \frac{1}{n^{s}},
$$

а в остальном - аналитическим продолжением, и пусть $Z$ есть функция Харди, определяемая равенством

$$
Z(t)=e^{i \theta(t)} \zeta\left(\frac{1}{2}+i t\right)
$$

где

$$
\theta(t)=\arg \left(\pi^{-i t / 2} \Gamma\left(\frac{1}{4}+i \frac{t}{2}\right)\right),
$$

а аргумент определяется непрерывным варьированием $t$, начиная со значения 0 при $t=0$. Можно показать (см. [1]), что

$$
\theta(t)=\frac{t}{2} \log \frac{t}{2 \pi}-\frac{t}{2}-\frac{\pi}{8}+O\left(\frac{1}{t}\right)
$$

Вещественные нули $Z$ совпадают с нулями $\zeta$, расположенными на прямой с вещественной частью $1 / 2$. Если гипотеза Римана верна, то для числа нулей $Z$ на интервале ]0, $t]$ справедливо соотношение (см. [1])

$$
N(t)=\frac{1}{\pi} \theta(t)+1+S(t)
$$

где

$$
S(t)=\frac{1}{\pi} \arg \zeta\left(\frac{1}{2}+i t\right)
$$

если $t$ не является нулем $Z$, и $\arg \zeta(1 / 2+i t)$ определяется непрерывной вариацией вдоль прямых соединяющих $2,2+i t$ и $1 / 2+i t$, начиная с исходного значений $\arg \zeta(2)=0$. Если $t-$ это нуль $Z$, положим

$$
S(t)=\lim _{\epsilon \rightarrow 0_{+}} S(t+\epsilon) .
$$

Известно (см. [1]), что если гипотеза Римана верна, то

$$
S(t)=O\left(\frac{\log t}{\log \log t}\right),
$$

но предполагается, что

$$
S(t)=O\left((\log t)^{1 / 2+o(1)}\right) .
$$


В настоящей статье мы получим при некоторых условиях оценку

$$
S(t)=O\left((\log t)^{1 / 2} \log \log \log t\right) .
$$

Поводом к нашему исследованию послужила захватывающая статья Ивича [2], в которой автор дает среди прочего условное опровержение гипотезы Римана, применяя производные $Z$ высокого порядка.

В настоящей статье используются стандартные обозначения: $\lfloor x\rfloor$ и $\lceil x\rceil$ для обычных функций антье и потолка (округление до ближайшего целого в большую сторону) и $\{x\}:=x-\lfloor x\rfloor$. Запись $f(x) \ll g(x)$ означает, что существуют $x_{0}$ и положительная константа $C$ такие, что $|f(x)| \leqslant C g(x)$ при $x \geqslant x_{0}$, где $g(x)$ положительна при $x \geqslant x_{0}$; аналогично $f(x) \gg g(x)$ и $f(x) \asymp g(x)$ означает, что $g(x) \ll f(x) \ll g(x)$. Многочлены Бернулли и Чебышёва степени $n$ обозначаются через $B_{n}(x)$ и $T_{n}(x)$ соответственно и определяются как

$$
\int_{x}^{x+1} B_{n}(t) d t=x^{n} \quad \text { и } \quad T_{n}(\cos \theta)=\cos n \theta .
$$

Наконец, при $r \geqslant 1$ и $t \geqslant t_{r}$ положим $\log _{1} t \equiv \log t$ и $\log _{r} t=\log \left(\log _{r-1} t\right)$ при $r \geqslant 2$.

Статья организована следующим образом: в п. 2 представлены основные результаты. Пункт 3 посвящен ключевому тождеству, доказанному автором в [3], и предварительным леммам. Доказательства теорем даны в п. 4.

В настоящей статье мы предполагаем справедливой гипотезу Римана и для простоты представления предполагаем также, что нули $\zeta$ являются простыми.

\section{2. Основные результаты}

В вычислениях, проведенных в [3], мы обнаружили, что функции $(-1)^{k+1} Z^{(2 k-1)}$ из [3] имеют, вообще говоря, те же знаки, и поступая как в [4] можно показать, что если $t_{n}$ таково, что $\theta\left(t_{n}\right)=(n+1 / 2) \pi$, то для достаточно больших $n$ числа $(-1)^{k+1} Z^{(2 k-1)}\left(t_{n}\right)$ имеют одинаковые знаки при $k \in\left[(3 / 4) \theta^{\prime}\left(t_{n}\right),(7 / 8) \theta^{\prime}\left(t_{n}\right)\right]$. Несмотря на то, что это не используется в наших доказательствах, это показывает, что производные высокого порядка $Z$ имеют правильное поведение.

Теорема 2.1. Пусть $T$ достаточно велико, $(\log T)^{-1 / 2} \ll a \ll(\log \log T)^{-1}$ такое, что $Z(T \pm a) \neq 0, u$

$$
(-1)^{k+1} Z^{(2 k-1)}(T \pm a) \geqslant 0 \quad \text { npu } \quad k=1,2, \ldots,\left\lceil\frac{\theta^{\prime}(T)}{4}\right\rceil .
$$

Далее, предполагаем, что существует положительная постоянная с такая, что $Z^{\prime}(T \pm a) \gg$ $\theta^{\prime}(T)^{-c}$. Тогда

$$
S(T+a)-S(T-a) \leqslant 2+\frac{4 \log 2}{\pi^{2}} a^{2} \log T(1+o(1)) .
$$

Из доказательства этой теоремы следует, что ее утверждение справедливо, если заменить неравенства (1) одним неравенством, включающим линейную комбинацию производных $Z$ нечетного порядка, вычисленных в $T \pm a$. Чтобы увидеть, что достаточные условия (1) могут быть удовлетворены, когда $S(T+a)-S(T-a)$ "большое", имеет смысл построить графики функций $S(T+t)$ и

$$
f_{2 k-1}(t):=(-1)^{k+1} \frac{Z^{(2 k-1)}(T+t)}{\theta^{\prime}(T)^{2 k-1}}
$$

для значений $T$, расположенных в окрестности точек, в которых $S$ "большое", и при $k=$ $1, \ldots,\left\lceil\theta^{\prime}(T) / 4\right\rceil$. Заметим, что член $\theta^{\prime}(T)^{-(2 k-1)}-$ это просто масштабный коэффициент. На 
рис. 1 и 2 эти графики изображены для значения $T=7.1934200352263673 \times 10^{14}$, для которого

$$
S(T+0.46009)=2.545 \ldots, \quad Z(T+0.9443)=738.756 \ldots, \quad\left\lceil\frac{\theta^{\prime}(T)}{4}\right\rceil=5 .
$$

Другой выбор $T$ порождает похожие графики.

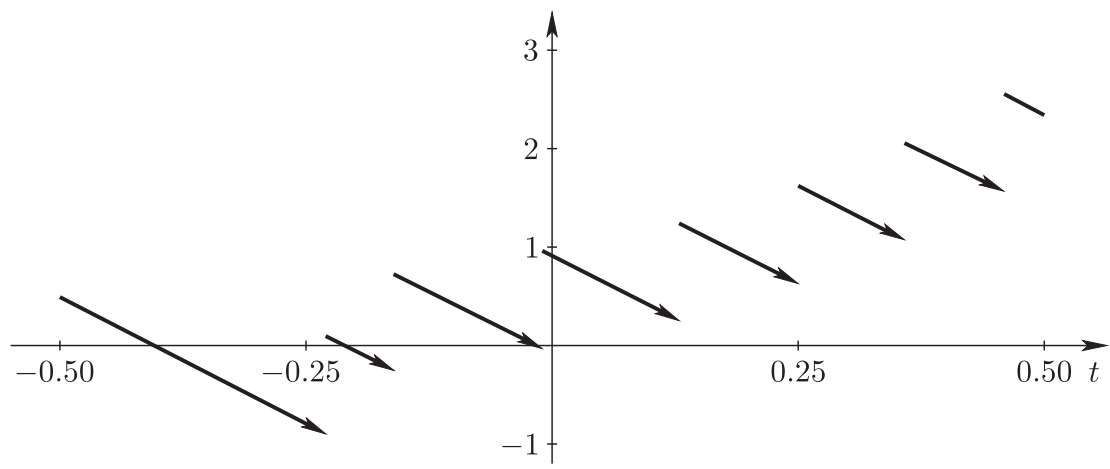

Рис. 1. График $S(T+t)$
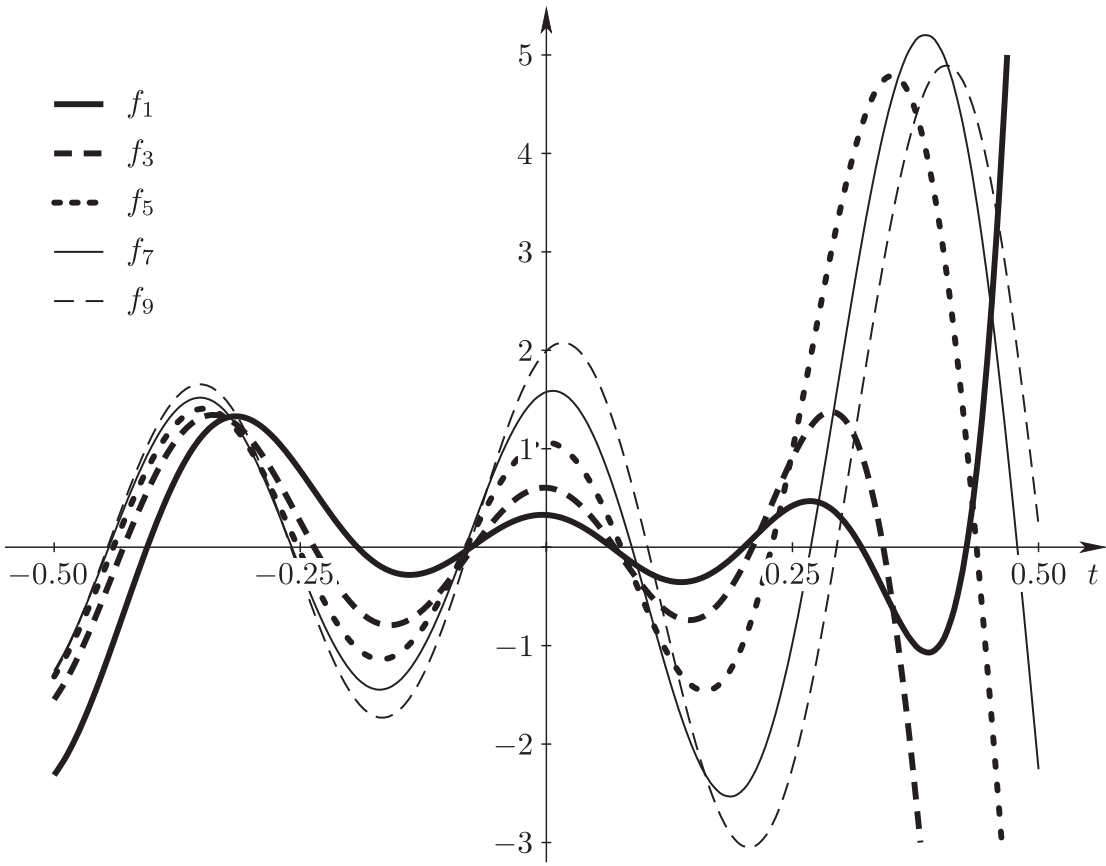

Рис. 2. Графики $f_{2 k-1}$

ТЕОРема 2.2. Пусть существуют последовательность $\left(T_{j}\right)$ и положительная констанmа с такие, что $T_{j+1}-T_{j} \asymp\left(\log T_{j}\right)^{-1 / 2}, Z\left(T_{j}\right) \neq 0$ u $Z^{\prime}\left(T_{j}\right) \gg \theta^{\prime}\left(T_{j}\right)^{-c}$ nри $j=0,1, \ldots$. Пусть, далее,

$$
(-1)^{k+1} Z^{(2 k-1)}\left(T_{j}\right) \geqslant 0 \quad \text { npu } \quad k=1,2, \ldots,\left\lceil\frac{\theta^{\prime}\left(T_{j+1}\right)}{4}\right\rceil, \quad j=0,1, \ldots .
$$


Тогда

$$
S(T)=O\left((\log T)^{1 / 2} \log _{3} T\right) .
$$

Заметим, что (2) соответствует гипотезе Монтгомери [5], которая утверждает, что $S(T)=$ $O\left((\log T)^{1 / 2}\left(\log _{2} T\right)^{-1 / 2}\right)$. Также уместно процитировать статью Мозера [6], на которую было указано рецензентом и в которой автор доказывает, что если число пар последовательных ординат $\gamma_{n}, \gamma_{n+1}$ нулей $\zeta$, расположенных на ] $T, 2 T[$ и удовлетворяющих неравенству

$$
0<\gamma_{n+1}-\gamma_{n} \leqslant \frac{4 \pi^{2}}{(\log T)^{2}}
$$

есть $o(T \log T)$, то для положительной доли интервалов $] \gamma_{n}, \gamma_{n+1}[\subset] T, 2 T[$ имеем $S(t)=$ $O\left((\log T)^{1 / 2}\left(\log _{2} T\right)^{2 / 3}\right)$ для любого $\left.t \in\right] \gamma_{n}, \gamma_{n+1}[$.

\section{3. Предварительные результаты}

Ключом к нашей аргументации является соотношение, которое связывает значение функции $f \in C^{2 r}[-a, a]$ в $x_{0}$, нули $f$ и значения ее производных нечетного порядка на границах интервала.

Лемма 3.1. Пусть $\left.r \in \mathbb{N}^{*}, a>0, f \in C^{2 r}[-a, a] u x_{0} \in\right]-a, a\left[\right.$ maкое, что $f\left(x_{0}\right) \neq 0$. Предположим, что $f$ имеет только конечное число нулей в $]-a, a[$, все они простые, обозначаемые $x_{k}$ с $k \neq 0$ и так пронумерованные, что $-a<x_{-m}<\cdots<x_{-1}<x_{0}<x_{1}<\cdots<x_{n}<a$. Далее, при $l \geqslant 1$ пусть $\Psi_{2 l-1}$ определяется как

$$
\Psi_{2 l-1}(x)=\frac{(4 a)^{2 l-1}}{(2 l) !} \sum_{k=-m}^{n} \mu_{k}\left(B_{2 l}\left(\frac{1}{2}+\frac{x+x_{k}}{4 a}\right)+B_{2 l}\left(\left\{\frac{x-x_{k}}{4 a}\right\}\right)\right),
$$

где $\mu_{k}=\alpha_{k} / \alpha_{0} u$

$$
\alpha_{k}=\frac{1}{\prod_{\substack{m \leqslant j \leqslant n \\ j \neq k}}\left(\sin \left(\pi \frac{x_{k}}{2 a}\right)-\sin \left(\pi \frac{x_{j}}{2 a}\right)\right)} \quad \text { npu } \quad k=-m, \ldots, n .
$$

Тогда

$$
f\left(x_{0}\right)=\sum_{k=1}^{r} \Psi_{2 k-1}(a) f^{(2 k-1)}(a)-\sum_{k=1}^{r} \Psi_{2 k-1}(-a) f^{(2 k-1)}(-a)-\int_{-a}^{a} \Psi_{2 r-1}(x) f^{(2 r)}(x) d x .
$$

Мы доказали в [3], что тождество (4) справедливо для функций $\Psi_{2 l-1}$, определяемых равенством (3), единственно лишь условиями $\mu_{0}=1$ и $\sum_{-m}^{n} \mu_{k}=0$. Наш особый выбор коэффициентов $\mu_{k}$ имеет решающее значение для получения хороших свойств функции $\Psi_{2 l-1}$, что станет понятным при доказательстве леммы 3.6. В [3] мы доказали также, что лемма 3.1 распространима на кратные нули.

ЛЕмма 3.2. Пусть $\Psi_{2 l-1}$ функиия, определяемая равенством (3), и пусть

$$
\bar{t}=\frac{1}{m+n+1} \sum_{k=-m}^{n} \sin \left(\pi \frac{x_{k}}{2 a}\right) .
$$

Тогда

$$
(-1)^{n+l+1} \Psi_{2 l-1}(a)>0 \quad u \quad(-1)^{m+l+1} \Psi_{2 l-1}(-a)>0 .
$$


Кроме того,

$$
\begin{gathered}
(-1)^{n} \Psi_{1}(a)>\frac{2 a(1+\bar{t})^{m+n}}{3\left|\alpha_{0}\right| \pi^{2}(m+n)^{2}}, \\
(-1)^{m} \Psi_{1}(-a)>\frac{2 a(1-\bar{t})^{m+n}}{3\left|\alpha_{0}\right| \pi^{2}(m+n)^{2}} .
\end{gathered}
$$

Для доказательства леммы 3.2 требуются два технических результата, полученные в леммах 3.3 и 3.4.

Лемма 3.3. Для всех $l, k \in \mathbb{N}^{*} u x \in[0,1[$ имеем неравенства

$$
\begin{aligned}
(-1)^{l+1} \frac{d^{k}}{d x^{k}} B_{2 l}\left(\frac{1}{2}+\frac{1}{\pi} \operatorname{Arcsin} \sqrt{x}\right) & >0, \\
\frac{1}{k !} \frac{d^{k}}{d x^{k}} B_{2}\left(\frac{1}{2}+\frac{1}{\pi} \operatorname{Arcsin} \sqrt{x}\right) & >\frac{2^{2 k}}{6 \pi^{2} k^{2}} x^{k} .
\end{aligned}
$$

ДокАзАтельство. Первое неравенство доказано в [3]. Для получения второго утверждения, заметим, что $B_{2}(1 / 2+x)=-1 / 12+x^{2}$,

$$
B_{2}\left(\frac{1}{2}+\frac{1}{\pi} \operatorname{Arcsin} \sqrt{x}\right)=-\frac{1}{12}+\frac{1}{\pi^{2}} \sum_{j=1}^{\infty} \frac{2^{2 j}((j-1) !)^{2}}{2(2 j) !} x^{j}
$$

и, следовательно, имеем

$$
\frac{1}{k !} \frac{d^{k}}{d x^{k}} B_{2}\left(\frac{1}{2}+\frac{1}{\pi} \operatorname{Arcsin} \sqrt{x}\right)=\frac{1}{\pi^{2}} \sum_{j=k}^{\infty} \frac{2^{2 j}((j-1) !)^{2} j !}{2(2 j) !(j-k) ! k !} x^{j-k}>c_{k} \frac{2^{2 k}}{\pi^{2} k^{2}} x^{k},
$$

где сумма тривиально оценивается снизу членом с индексом $j=2 k$ и

$$
c_{k}=\frac{2^{2 k}((2 k-1) !)^{2}(2 k) !}{2(4 k) !((k-1) !)^{2}} .
$$

Далее, замечаем, что

$$
\frac{c_{k+1}}{c_{k}}=\frac{(4 k+2)^{2}}{(4 k+2)^{2}-1}>1,
$$

откуда следует, что $c_{k}>c_{1}=1 / 6$, и мы завершаем доказательство, пользуясь (8).

Лемма 3.4. Пусть $I=]-a, a\left[\right.$, функиия $f \in C^{m+n+2}(I)$ maкая, что $f^{(m+n+2)}>0$ на $I, u$ пусть $t_{-m}, \ldots, t_{n} \in I$ - попарно различные числа. Тогда

$$
\sum_{k=-m}^{n} \frac{f\left(t_{k}\right)}{\prod_{\substack{m \leqslant j \leqslant n \\ j \neq k}}\left(t_{k}-t_{j}\right)}>\frac{f^{(m+n)}(\bar{t})}{(m+n) !}
$$

где $\bar{t}=\left(\sum_{-m}^{n} t_{k}\right) /(m+n+1)$.

ДокАЗАТЕЛьство. Это утверждение - непосредственное следствие результата Флоатера [7], который утверждает, что существует $\eta \in\left[t_{-m}, t_{n}\right]$ такое, что

$$
\sum_{k=-m}^{n} \frac{f\left(t_{k}\right)}{\prod_{\substack{m \leqslant j \leqslant n \\ j \neq k}}\left(t_{k}-t_{j}\right)}=\frac{f^{(m+n)}(\bar{t})}{(m+n) !}+R_{m+n+2},
$$


где

$$
R_{m+n+2}=\frac{1}{2(m+n+1)}\left(\sum_{-m \leqslant i<j \leqslant n}\left(t_{j}-t_{i}\right)^{2}\right) \frac{f^{(m+n+2)}(\eta)}{(m+n+2) !} .
$$

ДоказАТЕЛьСтво ЛЕммы 3.2. Неравенства (5) доказаны в работе [3]. Поскольку $B_{2}(x)=$ $B_{2}(1-x)$, имеем

$$
\Psi_{1}( \pm a)=4 a \sum_{k=-m}^{n} \mu_{k} B_{2}\left(\frac{3}{4} \pm \frac{x_{k}}{4 a}\right)
$$

и, следовательно,

$$
(-1)^{n} \Psi_{1}( \pm a)=\left(\frac{(-1)^{n}}{\alpha_{0}}\right) 4 a \sum_{k=-m}^{n} \alpha_{k} h_{ \pm}\left(t_{k}\right),
$$

где $h_{ \pm}(t)=B_{2}(3 / 4 \pm(1 /(2 \pi)) \operatorname{Arcsin} t)$, и $t_{k}=\sin \left(\pi x_{k} /(2 a)\right)$. Первый член в правой части положителен, и тождества

$$
\frac{3}{4} \pm \frac{1}{2 \pi} \operatorname{Arcsin} t=\frac{1}{2}+\frac{1}{\pi} \operatorname{Arcsin} \sqrt{\frac{1 \pm t}{2}} \quad \text { при } \quad t \in[-1,1]
$$

вместе с леммой 3.3 показывают, что $h_{+}^{(m+n+2)}$ и $(-1)^{(m+n)} h_{-}^{(m+n+2)}$ положительны на ]-1, 1[. Доказательство (6) и (7) завершается использованием леммы 3.4 и леммы 3.3 для вычисления нижних оценок для $h_{+}^{(m+n)}(\bar{t})$ и $(-1)^{(m+n)} h_{-}^{(m+n)}(\bar{t})$.

Лемма 3.5. Пусть $b_{r, s}-$ числа, определенные для цельх $r \geqslant 3 u s \geqslant 0$ как

$$
b_{r, s}=\left(\frac{r}{r+s}\right)^{2 r \log r / \log _{2} r-1}\left(\begin{array}{c}
2 r+s-1 \\
s
\end{array}\right) .
$$

Тогда

$$
\sum_{s=0}^{\infty} b_{r, s}^{2}=e^{o(r)} \quad \text { npu } \quad r \rightarrow \infty .
$$

ДокАЗАТЕЛЬСтво. Так как $b_{r, s}=O_{r}\left(s^{-2 r \log r / \log _{2} r+2 r}\right)=O_{r}\left(s^{-1}\right)$, эта сумма сходится. Пользуясь формулой $\log n !=n \log n-n+O(\log n)$ и полагая $p=2 r \log r / \log _{2} r-1$, имеем $\log b_{r, s}=\phi_{r}(s)+O(\log r)$ при $1 \leqslant s \leqslant r$, где $\phi_{r}$ есть функция, определенная на отрезке $[1, r]$ каK

$$
\phi_{r}(x)=-p \log \left(1+\frac{x}{r}\right)+(2 r+x-1) \log (2 r+x-1)-x \log x-(2 r-1) \log (2 r-1) .
$$

При достаточно больших $r$ проверяется, что $\phi_{r}^{\prime}(1)>0, \phi_{r}^{\prime}(r)<0$ и $\phi_{r}^{\prime \prime}$ имеет единственную перемену знака, которая подразумевает, что $\phi_{r}$ имеет единственный максимум, расположенный в $\left.x_{r}^{*} \in\right] 1, r\left[\right.$. Переписав уравнение $\phi_{r}^{\prime}(x)=0$ в виде

$$
x=2 r e^{-p / r}\left(e^{p x /(r(r+x))}\left(1+\frac{x}{2 r}-\frac{1}{2 r}\right)\right),
$$

заключаем, что

$$
x_{r}^{*}=2 r^{1-2 / \log _{2} r}\left(1+O\left(r^{-1 / \log _{2} r}\right)\right), \quad \phi_{r}\left(x_{r}^{*}\right)=2 r^{1-2 / \log _{2} r}\left(1+O\left(r^{-1 / \log _{2} r}\right)\right),
$$

откуда

$$
\sum_{s=1}^{r} b_{r, s}^{2} \leqslant r e^{2 \phi_{r}\left(x_{r}^{*}\right)+O(\log r)}=e^{o(r)}
$$


При $s \geqslant r$ введем числа $c_{r, s}:=b_{r, s+1} / b_{r, s}$ и проверим, что

$$
s \log c_{r, s}=-s p \log \left(1+\frac{1}{r+s}\right)+s \log \left(1+\frac{2 r-1}{s+1}\right) \leqslant-s p\left(\frac{1}{r+s}-\frac{1}{2} \frac{1}{(r+s)^{2}}\right)+2 r .
$$

Замечая, что последнее выражение убывает с $s$, выбираем $s=r$, чтобы получить неравенство

$$
s \log c_{r, s} \leqslant-p\left(\frac{1}{2}-\frac{1}{8 r}\right)+2 r \leqslant-1 .
$$

Отсюда следует, что

$$
\frac{b_{r, s+1}}{b_{r, s}} \leqslant e^{-1 / s} \leqslant \frac{s}{s+1},
$$

что подразумевает

$$
b_{r, s} \leqslant \frac{r b_{r, r}}{s} \quad \text { при } \quad s=r, r+1, \ldots
$$

а поскольку $b_{r, r} \leqslant e^{\phi_{r}\left(x_{r}^{*}\right)+O(\log r)}$, мы заключаем, что $\sum_{s=r+1}^{\infty} b_{r, s}^{2}=e^{o(r)}$.

Лемма 3.6. Пусть $\Psi_{2 l-1}$ есть функиия, определяемая (3), пусть $m+n$ достаточно большое $и l \geqslant(m+n) \log (m+n) / \log _{2}(m+n)$. Тогда

$$
\left\|\Psi_{2 l-1}\right\|_{2} \leqslant \frac{2^{(m+n)(1+o(1))}}{\left|\alpha_{0}\right| \sqrt{a}}\left(\frac{2 a}{(m+n) \pi}\right)^{2 l} \quad \text { npu } \quad m+n \rightarrow \infty,
$$

где

$$
\left\|\Psi_{2 l-1}\right\|_{2}^{2}=\int_{-a}^{a}\left(\Psi_{2 l-1}(x)\right)^{2} d x .
$$

ДокАзАТЕЛьство. Применяя разложение в ряд Фурье

$$
B_{2 l}(x)=(-1)^{l+1} 2((2 l) !) \sum_{j=1}^{\infty} \frac{1}{(2 j \pi)^{2 l}} \cos (2 j \pi x) \quad \text { для } \quad x \in[0,1]
$$

и тождество

$$
\cos \alpha+\cos \beta=2 \cos \left(\frac{\alpha+\beta}{2}\right) \cos \left(\frac{\alpha-\beta}{2}\right)
$$

приходим к выражению

$$
\Psi_{2 l-1}(x)=(-1)^{l+1} 2 \frac{(2 a)^{2 l-1}}{\alpha_{0} \pi^{2 l}} \sum_{j=1}^{\infty} \frac{1}{j^{2 l}}\left(\sum_{k=-m}^{n} \alpha_{k} \cos \left(j \pi\left(\frac{1}{2}+\frac{x_{k}}{2 a}\right)\right)\right) \cos \left(j \pi\left(\frac{1}{2}+\frac{x}{2 a}\right)\right) .
$$

Пользуясь тождеством

$$
\cos \left(j \pi\left(\frac{1}{2}+y\right)\right)=(-1)^{j} T_{j}(\sin (\pi y))
$$

и полагая

$$
a_{j, k}=(-1)^{j} T_{j}\left(\sin \left(\pi \frac{x_{k}}{2 a}\right)\right)
$$

имеем

$$
\sum_{k=-m}^{n} \alpha_{k} a_{j, k}=0 \quad \text { при } \quad j=1, \ldots, m+n-1 .
$$

Последнее обосновывает наш особый выбор $\alpha_{k}$ при определении $\Psi_{2 l-1}$. Отсюда имеем

$$
\Psi_{2 l-1}(x)=(-1)^{l+1} 2 \frac{(2 a)^{2 l-1}}{\alpha_{0} \pi^{2 l}} \sum_{j=m+n}^{\infty} \frac{1}{j^{2 l}}\left(\sum_{k=-m}^{n} \alpha_{k} a_{j, k}\right) \cos \left(j \pi\left(\frac{1}{2}+\frac{x}{2 a}\right)\right) .
$$


Применяя стандартный результат, касающийся разделенных разностей, возводя в квадрат (9) и интегрируя по отрезку $[-a, a]$, получаем

$$
\left\|\Psi_{2 l-1}\right\|_{2}^{2}=\left(2 \frac{(2 a)^{2 l-1}}{\alpha_{0} \pi^{2 l}}\right)^{2} \sum_{j=m+n}^{\infty} \frac{1}{j^{4 l}}\left(\frac{T_{j}^{(m+n)}\left(\tau_{j}\right)}{(m+n) !}\right)^{2} a
$$

для некоторых $\left.\tau_{j} \in\right]-1,1[$. Известно (см. [8]), что

$$
\max _{-1 \leqslant x \leqslant 1}\left|T_{j}^{(m+n)}(x)\right|=T_{j}^{(m+n)}(1)=2^{m+n-1}(m+n-1) ! j\left(\begin{array}{c}
m+n+j-1 \\
j-m-n
\end{array}\right)
$$

при $j=m+n, m+n+1, \ldots$, и, следовательно,

$$
\left\|\Psi_{2 l-1}\right\|_{2}^{2} \leqslant\left(\frac{2^{m+n-1}}{\alpha_{0} \sqrt{a}}\left(\frac{2 a}{(m+n) \pi}\right)^{2 l}\right)^{2} \sum_{j=m+n}^{\infty}\left(\frac{m+n}{j}\right)^{4 l-2}\left(\begin{array}{c}
m+n+j-1 \\
j-m-n
\end{array}\right)^{2} .
$$

Положим $j=m+n+s$, а так как $l \geqslant(m+n) \log (m+n) / \log _{2}(m+n)$, имеем

$$
\begin{aligned}
\left\|\Psi_{2 l-1}\right\|_{2}^{2} & \leqslant\left(\frac{2^{m+n-1}}{\alpha_{0} \sqrt{a}}\left(\frac{2 a}{(m+n) \pi}\right)^{2 l}\right)^{2} \sum_{s=0}^{\infty}\left(\left(\frac{m+n}{m+n+s}\right)^{2 l-1}\left(\begin{array}{c}
2(m+n)+s-1 \\
s
\end{array}\right)\right)^{2} \\
& \leqslant\left(\frac{2^{m+n-1}}{\alpha_{0} \sqrt{a}}\left(\frac{2 a}{(m+n) \pi}\right)^{2 l}\right)^{2} \sum_{s=0}^{\infty} b_{m+n, s}^{2}
\end{aligned}
$$

и мы завершаем доказательство, пользуясь леммой 3.5.

\section{4. Доказательства теорем}

Для достаточно большого $T$ и для $(\log T)^{-1 / 2} \ll a \ll(\log \log T)^{-1}$ такого, что $Z(T \pm a) \neq 0$ и $\Delta S:=S(T+a)-S(T-a) \geqslant 0$, существуют $\left.t_{0} \in\right] T-a, T+a[$ и положительные целые числа $m$ и $n$ (четное и нечетное соответственно) такие, что $Z\left(t_{0}\right)>0$ и

$$
\gamma_{-m-2}<T-a<\gamma_{-m}<\cdots<\gamma_{-1}<t_{0}<\gamma_{1}<\cdots<\gamma_{n}<T+a<\gamma_{n+2}
$$

где $\gamma_{k}$ - нули $Z$. Следует отметить, что мы не принимаем во внимание нули $\gamma_{-m-1}$ и $\gamma_{n+1}$, даже если они принадлежат отрезку $[T-a, T+a]$. Поэтому

$$
m+n=\frac{1}{\pi}(\theta(T+a)-\theta(T-a))+\Delta S^{*},
$$

где $\Delta S^{*}=\Delta S-q$ и $q=0,1,2$ в соответствии с положением $\gamma_{-m-1}$ и $\gamma_{n+1}$ относительно $T-a$ и $T+a$. Тождество (4) записывается как

$$
\left.Z\left(t_{0}\right)=\sum_{k=1}^{K} \Psi_{2 k-1}(a) Z^{(2 k-1)}(T+a)-\sum_{k=1}^{K} \Psi_{2 k-1}(-a) Z^{(2 k-1)}(T-a)\right)+R,
$$

где

$$
R=-\int_{-a}^{a} \Psi_{2 K-1}(x) Z^{(2 K)}(T+x) d x
$$

и $x_{k}$, которые неявно используются в определении $\Psi_{2 l-1}$, определяются как $x_{0}=t_{0}-T$ и $x_{k}=\gamma_{k}-T$ при $k \neq 0$. 
Лемма 4.1. Пусть $T$ достаточно велико, пусть $(\log T)^{-1 / 2} \leqslant a \leqslant(\log \log T)^{-1} u K=$ $\left\lceil\theta^{\prime}(T) / 4\right\rceil$. Предположим, далее, что $\Delta S^{*} \geqslant 0$. Тогда

$$
|R| \leqslant \frac{1}{\left|\alpha_{0}\right|} \exp \left(\left(\left(\frac{2 a}{\pi} \theta^{\prime}(T)+\Delta S^{*}\right) \log 2\right)(1+o(1))-\frac{\theta^{\prime}(T)}{2} \log \left(1+\pi \frac{\Delta S^{*}}{2 a \theta^{\prime}(T)}\right)\right) .
$$

Доказательство этой оценки требует следующей теоремы, доказанной автором в [4], которая справедлива без предположения справедливости гипотезы Римана.

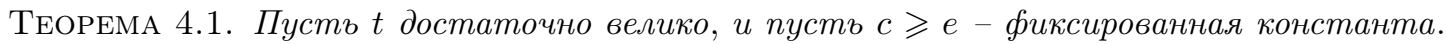
Тогда при $0 \leqslant k \leqslant(c / 2) \theta^{\prime}(t)$ имеем равномерно по $k$

$$
Z^{(k)}(t)=2 \sum_{1 \leqslant n \leqslant \sqrt{t /(2 \pi)}} \frac{1}{\sqrt{n}}\left(\theta^{\prime}(t)-\log n\right)^{k} \cos \left(\theta(t)-t \log n+k \frac{\pi}{2}\right)+O\left(t^{(1 / 4)(c \log c-c-1)} \theta^{\prime}(t)^{k+1}\right) .
$$

ДокАЗАТЕЛЬСТВо ЛЕммы 4.1. При $t \in[T-a, T+a]$ с учетом $2 K \leqslant \theta^{\prime}(T) / 2+2$ и $\theta^{\prime \prime}(t)=$ $O(1 / t)$ можно воспользоваться теоремой 4.1 с $c=e$ для получения оценки

$$
\left|Z^{(2 K)}(t)\right| \leqslant\left(2\left(\sum_{1 \leqslant n \leqslant \sqrt{t /(2 \pi)}} \frac{1}{\sqrt{n}}\left(1-\frac{\log n}{\theta^{\prime}(t)}\right)^{2 K}\right)+O\left(t^{-1 / 4} \theta^{\prime}(t)\right)\right) \theta^{\prime}(t)^{2 K} .
$$

Кроме того, $2 K \geqslant \theta^{\prime}(T) / 2, \theta^{\prime}(T) / \theta^{\prime}(t)=1+O(a / T)$ и, следовательно, имеют место неравенства

$$
\left(1-\frac{\log n}{\theta^{\prime}(t)}\right)^{2 K} \leqslant e^{-2 K \log n / \theta^{\prime}(t)} \leqslant n^{-1 / 2+O(a / T)} \leqslant 2 n^{-1 / 2}
$$

при $1 \leqslant n \leqslant \sqrt{t / 2 \pi}$ и достаточно большом $T$. Отсюда следует, что

$$
\left|Z^{(2 K)}(t)\right| \leqslant\left(4\left(\sum_{1 \leqslant n \leqslant \sqrt{t /(2 \pi)}} \frac{1}{n}\right)+O\left(t^{-1 / 4} \theta^{\prime}(t)\right)\right) \theta^{\prime}(t)^{2 K} \leqslant 4(\log T) \theta^{\prime}(T)^{2 K} .
$$

Пользуясь формулой Тейлора и оценкой $\theta^{\prime \prime \prime}(t)=O\left(1 / t^{2}\right)$, имеем

$$
m+n=\frac{2 a}{\pi} \theta^{\prime}(T)+\Delta S^{*}+O\left(\frac{a^{3}}{T^{2}}\right) .
$$

Отсюда $K \geqslant(m+n) \log (m+n) / \log _{2}(m+n)$, так как $\Delta S^{*}=O\left(\log T / \log _{2} T\right)$, если гипотеза Римана верна. Поэтому применяется лемма 3.6 и, пользуясь неравенством Коши-Шварца, получаем

$$
|R| \leqslant 4 \sqrt{2} \log T \frac{2^{(m+n)(1+o(1))}}{\left|\alpha_{0}\right|} \exp \left(-2 K \log \left(\frac{(m+n) \pi}{2 a \theta^{\prime}(T)}\right)\right) .
$$

Для завершения доказательства воспользуемся (12) и заметим, что $K O\left(a^{2} /\left(\theta^{\prime}(T) T^{2}\right)\right)$ есть $o\left(a \theta^{\prime}(T)\right)$ и $4 \sqrt{2} \log T$ может быть покрыто членом $e^{(2 a / \pi) \theta^{\prime}(T) o(1)}$.

Лемма 4.2. Для достаточно малого $\epsilon>0$ допустим, что $x \geqslant 0$ u $x=o(1)$ nри $\epsilon$, cmpeмящемся $\kappa 0, u$

$$
(\epsilon+x) \log 2+o(\epsilon)+o(x)-\frac{1}{2} \log \left(1+\frac{x}{\epsilon}\right) \geqslant 0 .
$$

Тогда $x \in\left[0,(2 \log 2) \epsilon^{2}+o\left(\epsilon^{2}\right)\right]$. 
ДоКАЗАТЕЛЬСТВо теОРЕмЫ 2.1. Пусть $T$ достаточно велико, и пусть $a, K, \Delta S^{*}, t_{0}, m$ и $n$ такие, какими определены в начале пункта. Заметим, что

$$
S(T+a)-S(T-a)-2=\Delta S^{*}+q-2 \leqslant \Delta S^{*},
$$

и, значит, теорема 2.1 верна, если $\Delta S^{*} \leqslant 0$. Следовательно, можно предположить, что $\Delta S^{*}>0$, так что применима лемма 4.1. Из тождества (10) следует, что

$$
R>-\sum_{k=1}^{K} \Psi_{2 k-1}(a) Z^{(2 k-1)}(T+a)+\sum_{k=1}^{K} \Psi_{2 k-1}(-a) Z^{(2 k-1)}(T-a),
$$

и из (1) и (5) мы получаем

$$
-\Psi_{2 k-1}(a) Z^{(2 k-1)}(T+a) \geqslant 0, \quad \Psi_{2 k-1}(-a) Z^{(2 k-1)}(T-a) \geqslant 0 \quad \text { при } \quad k=1,2, \ldots,\left\lceil\frac{\theta^{\prime}(T)}{4}\right\rceil .
$$

Выбирая $k=1$ и пользуясь (6) и (7) вместе с неравенством $(1+\bar{t})^{m+n}+(1-\bar{t})^{m+n} \geqslant 2$, получаем

$$
R \gg \frac{a \theta^{\prime}(T)^{-c}}{\left|\alpha_{0}\right|(m+n)^{2}},
$$

откуда заключаем в силу (11), что

$$
\left(\frac{2 a}{\pi} \theta^{\prime}(T)+\Delta S^{*}\right) \log 2+o\left(a \theta^{\prime}(T)\right)+o\left(\Delta S^{*}\right)-\frac{\theta^{\prime}(T)}{2} \log \left(1+\pi \frac{\Delta S^{*}}{2 a \theta^{\prime}(T)}\right) \geqslant 0 .
$$

Разделив на $\theta^{\prime}(T)$, полагая $\epsilon=2 a / \pi$ и $x=\Delta S^{*} / \theta^{\prime}(T)$ и замечая, что $x=o(1)$ при $\epsilon$, стремящемся к 0 , примененим лемму 4.2 и получим

$$
\Delta S^{*} \leqslant(2 \log 2) \epsilon^{2} \theta^{\prime}(T)(1+o(1))=2 \log 2\left(\frac{2 a}{\pi}\right)^{2} \theta^{\prime}(T)(1+o(1)) .
$$

Доказательство завершено, поскольку $\theta^{\prime}(T)=(1 / 2) \log T(1+o(1))$.

ДоКАЗАТЕЛЬСТВо тЕОРЕмЫ 2.2. Пусть $T$ достаточно велико, и предположим сначала, что $S(T)>0$. В соответствии с [9], если гипотеза Римана верна, существует $\underline{t}<T$ такое, что $S(\underline{t})<0$ и $T-\underline{t} \ll \log _{3} T$. По условию теоремы существуют целые $j<k$ такие, что

$$
T_{j}<\underline{t} \leqslant T_{j+1}<\cdots<T_{k-1} \leqslant T<T_{k},
$$

и вместе с тем $k-j=O\left((\log T)^{1 / 2} \log _{3} T\right)$. Отсюда заключаем, что

$$
\begin{aligned}
S(T) & \leqslant S(T)-S(\underline{t})=S\left(T_{j}\right)-S(\underline{t})+\left(\sum_{l=j}^{k-1} S\left(T_{l+1}\right)-S\left(T_{l}\right)\right)+S(T)-S\left(T_{k}\right) \\
& \leqslant \frac{1}{\pi}\left(\theta(\underline{t})-\theta\left(T_{j}\right)\right)+O\left((\log T)^{1 / 2} \log _{3} T\right)+\frac{1}{\pi}\left(\theta\left(T_{k}\right)-\theta(T)\right)=O\left((\log T)^{1 / 2} \log _{3} T\right),
\end{aligned}
$$

так как по теореме 2.1 существует константа $C$ такая, что $S\left(T_{l+1}\right)-S\left(T_{l}\right) \leqslant C$. Теперь если $S(T)<0$, то существуют $\bar{t}>T$ такое, что $S(\bar{t})>0$ и $\bar{t}-T \ll \log _{3} T$, и целые числа $j<k$ такие, что

$$
T_{j}<T \leqslant T_{j+1}<\cdots<T_{k-1} \leqslant \bar{t}<T_{k}
$$

и $k-j=O\left((\log T)^{1 / 2} \log _{3} T\right)$. Следовательно,

$$
\begin{aligned}
-S(T) & \leqslant-S(T)+S\left(T_{j}\right)+\left(\sum_{l=j}^{k-1} S\left(T_{l+1}\right)-S\left(T_{l}\right)\right)+S(\bar{t})-S\left(T_{k}\right) \\
& \leqslant \frac{1}{\pi}\left(\theta(T)-\theta\left(T_{j}\right)\right)+O\left((\log T)^{1 / 2} \log _{3} T\right)+\frac{1}{\pi}\left(\theta\left(T_{k}\right)-\theta(\bar{t})\right)=O\left((\log T)^{1 / 2} \log _{3} T\right),
\end{aligned}
$$

и доказательство завершено. 


\section{Список литературы}

[1] A. Ivić, The Theory of Hardy's Z-function, Cambridge Tracts in Math., 196, Cambridge Univ. Press, Cambridge, 2013.

[2] A. Ivić, "On some reasons for doubting the Riemann hypothesis", The Riemann Hypothesis. A Resource for the Afficionado and Virtuoso Alike, Springer, New York, 2008, 130-160.

[3] Ph. Blanc, "An unexpected property of odd order derivatives of Hardy's function", Publ. Inst. Math. (Beograd) (N.S.), 95 (109) (2014), 173-188.

[4] Ph. Blanc, "Optimal upper bound for the maximum of the $k$-th derivative of Hardy's function", J. Number Theory, 154 (2015), 105-117.

[5] H. L. Montgomery, "Extreme values of the Riemann zeta function", Comment. Math. Helv., 52:4 (1977), 511-518.

[6] J. Moser, "The function $S(t)$ in the theory of the Riemann zeta-function", Acta Arith., 30:2 (1976), $145-158$.

[7] M. S. Floater, "Error formulas for divided difference expansions and numerical differentiation", J. Approx. Theory, 122:1 (2003), 1-9.

[8] T. J. Rivlin, Chebyshev Polynomials. From Approximation Theory to Algebra and Number Theory, John Wiley and Sons, New York, 1990.

[9] J. Mueller, "On the Riemann zeta-function $\zeta(s)$-gaps between sign changes of $S(t)$ ", Mathematika, 29:2 (1983), 264-269. 